Subscriber access provided by UCL Library Services

Letter

\title{
Modulation of the allosteric communication between the polo- box domain and the catalytic domain in Plk1 by small compounds
}

Monika Raab, Mourad Sanhaji, Larissa Pietsch, Isabelle Béquignon, Amanda K

Herbrand, Evelyn Süß, Santosh L. Gande, Birgit Caspar, Denis Kudlinzki, Krishna

Saxena, Sridhar Sreeramulu, Harald Schwalbe, Klaus Strebhardt, and Ricardo M. Biondi

ACS Chem. Biol., Just Accepted Manuscript • DOI: 10.1021/acschembio.7b01078 • Publication Date (Web): 21 Jun 2018

Downloaded from http://pubs.acs.org on June 22, 2018

\section{Just Accepted}

"Just Accepted" manuscripts have been peer-reviewed and accepted for publication. They are posted online prior to technical editing, formatting for publication and author proofing. The American Chemical Society provides "Just Accepted" as a service to the research community to expedite the dissemination of scientific material as soon as possible after acceptance. "Just Accepted" manuscripts appear in full in PDF format accompanied by an HTML abstract. "Just Accepted" manuscripts have been fully peer reviewed, but should not be considered the official version of record. They are citable by the Digital Object Identifier (DOI $\left.{ }^{8}\right)$. "Just Accepted" is an optional service offered to authors. Therefore, the "Just Accepted" Web site may not include all articles that will be published in the journal. After a manuscript is technically edited and formatted, it will be removed from the "Just Accepted" Web site and published as an ASAP article. Note that technical editing may introduce minor changes to the manuscript text and/or graphics which could affect content, and all legal disclaimers and ethical guidelines that apply to the journal pertain. ACS cannot be held responsible for errors or consequences arising from the use of information contained in these "Just Accepted" manuscripts. 


\section{Modulation of the allosteric communication between the polo-box domain and the catalytic domain in Plk1 by small compounds}

Monika Raab ${ }^{2}$, Mourad Sanhaji ${ }^{2}$, Larissa Pietsch ${ }^{1,4}$, Isabelle Béquignon ${ }^{1}$, Amanda K. Herbrand ${ }^{1}$, Evelyn Süß ${ }^{1}$, Santosh L. Gande ${ }^{3,4}$, Birgit Caspar ${ }^{3}$, Denis Kudlinzki ${ }^{3,4}$, Krishna Saxena ${ }^{3}$, Sridhar Sreeramulu $^{3}$, Harald Schwalbe ${ }^{3,4}$, Klaus Strebhardt ${ }^{2,4}$, Ricardo M. Biondi ${ }^{1,4,5}$

${ }^{1}$ Research Group PhosphoSites, Medizinische Klinik 1, Universitätsklinikum Frankfurt, Frankfurt am Main, Germany.

${ }^{2}$ Department of Gynecology, Goethe-University, ${ }^{3}$ German Cancer Consortium (DKTK)

${ }^{3}$ Center for Biomolecular Magnetic Resonance, Johann Wolfgang Goethe University, 60438

Frankfurt, Germany

${ }^{4}$ German Cancer Consortium (DKTK), Heidelberg, Germany; German Cancer

Research Center (DKFZ), Heidelberg, Germany.

${ }^{5}$ Instituto de Investigación en Biomedicina de Buenos Aires (IBioBA) - CONICET - Partner

Institute of the Max Planck Society, Buenos Aires C1425FQD, Argentina

\section{Corresponding Authors:}

Ricardo M. Biondi

Research Group PhosphoSites

Medizinische Klinik 1

Universitätsklinikum Frankfurt

Theodor-Stern-Kai 7

60590 Frankfurt

Tel: +49696301 4591

FAX: +49696301 87689

e-mail: biondi@med.uni-frankfurt.de

Klaus Strebhardt

Department of Gynecology

Goethe-University

Theodor-Stern-Kai 7

60590 Frankfurt

Tel: +496963016894 


\author{
FAX: +496963016364 \\ e-mail: strebhardt@em.uni-frankfurt.de
}

Running title: Allosteric modulators of Plk1

\title{
Letter
}

\begin{abstract}
: 150 words
The Polo-like kinases (Plks) are an evolutionary conserved family of Ser/Thr protein kinases that possess, in addition to the classical kinase domain at the N-terminus, a C-terminal polo-box domain (PBD) that binds to phosphorylated proteins, modulates the kinase activity and its localization. Plk1, which regulates the formation of the mitotic spindle, has emerged as a validated drug target for the treatment of cancer, because it is required for numerous types of cancer cells but not for the cell division in non-cancer cells. Here, we employed chemical biology methods to investigate the allosteric communication between the PBD and the catalytic domain of Plk1. We identified small compounds that bind to the catalytic domain and inhibit or enhance the interaction of Plk1 with the phosphorylated peptide PoloBoxtide in vitro. In cells, two new allosteric Plk1 inhibitors affected the proliferation of cancer cells in culture and the cell cycle, but had distinct phenotypic effects on spindle formation. Both compounds inhibited Plk1 signaling indicating that they specifically act on Plk1 in cultured cells.
\end{abstract}




\section{Introduction}

Protein kinases are a large family of proteins comprising an evolutionary conserved catalytic domain ${ }^{l}$. Protein kinases function like molecular switches regulating a large set of signaling pathways ${ }^{2}$. The whole family has therefore evolved very precise and specific mechanisms to regulate their activities ${ }^{3}$, to properly transfer signals and to respond to the requirements of the cells at the appropriate cellular location and the right time. The Polo-like kinase 1 (Plk1) is conserved throughout eukaryotic organisms, locating during the cell division at the mitotic spindle triggering the $\mathrm{G}_{2} / \mathrm{M}$ phase of the cell cycle and participating in the segregation of chromosomes to the two daughter cells.

Human Plk1 comprises a catalytic domain followed by a polo box domain (PBD) (Figure 1A). Inhibitors of Plk1 cause cell cycle arrest at $\mathrm{G}_{2} / \mathrm{M}$ followed by the induction of apoptosis in a variety of cancer cell types. An increasing number of Plk inhibitors has been developed for the treatment of cancer patients. One ATP-binding site inhibitor, BI6727, received FDA Breakthrough Therapy designation for treatment of patients suffering from acute myeloid leukemia (AML). Since all protein kinases share similarities at the ATP-binding site, this type of inhibitor frequently inhibits many other protein kinases ${ }^{4}$, leading to off-target side effects in patients. Alternatively, targeting the mechanism of regulation of protein kinases can lead to very selective inhibitors that may be more suitable for combination therapies and long-term treatments.

Plks are regulated by PBD-mediated localization, by phosphorylation, by ubiquitination and by an intramolecular interaction between the catalytic domain and the PBD. The crystal structure of the catalytic domain has been solved in complex with ATP-competitive inhibitors, i.e. BI6727 5 and the PBD has been investigated by crystallography, thereby depicting the molecular details of the interaction with phosphorylated polypeptides ${ }^{6}$. More recently the structure of the Plk 1 from Danio rerio (zebrafish) showed how the PBD interacts and inhibits the catalytic domain ${ }^{7}$. Binding of phosphorylated polypeptides to the PBD both, regulates the subcellular localization / co-localization with substrates and activates the kinase domain ${ }^{8}$ (see scheme in Figure 1 B,C). Phosphorylation at T210 is also important for the regulation of the activity of Plk1. Low molecular weight inhibitors that bind to the PBD of Plk1 have been identified ${ }^{9-12}$. Together, the derived model of Plk1 regulation supports the existence of a dynamic allosteric mechanism of 
regulation which may be modulated by small compounds, as identified earlier in other protein kinases ${ }^{13,14}$. Here we use the terminology "allostery" and "allosteric" to refer to the biochemical phenomenon where the binding of a macromolecular partner or a small-molecule ligand at one location leads to specific perturbations at a site not in direct contact with the region where the binding occurs 15,16 . This definition differs from a frequent usage of the terminology in the protein kinase field where "allosteric" refers to a compound that binds at a site different from the ATP-binding site, independently of a known effect at a distant site.

In the present work we investigated the allosteric mechanism of Plk1 regulation using a chemical biology approach. We identified compounds that enhance or inhibit the interaction of Plk1 with the phosphorylated polypeptide that binds to the PBD. Two of the compounds, FM00204 and SCR01010, disrupted Plk1 localization and inhibited Plk1 function in cells. Our work describes aspects of the allosteric communication between the PBD and the catalytic domain of Plk1 in vitro and its effects in cells, indicating a fundamental role of the proper PBD-driven dynamic modulation of the conformation of Plk1 in cells and validating new allosteric drug development approaches to Plk1.

\section{Results and Discussion}

\section{Identification and in vitro characterization of small compound allosteric modulators of PIk1}

The intrinsic protein kinase activity of Plk1 can be measured using a polypeptide substrate (KKGGSFNDTLDFD) derived from Lansing et al. ${ }^{17}$. The assay allows measuring the intrinsic kinase activity of the catalytic domain of Plk1 and the effect of compounds that may affect the catalytic domain. In this in vitro assay, the characteristic phosphorylated polypeptide derived from the Plk1 substrate Cdc25 that binds to the PBD, PoloBoxtide (MAGPMQST(P)PLNGAK), activated Plk1 as described ${ }^{8}$ (Figure 1D).

To analyze the allosteric mechanisms that can modulate Plk1 conformation and activity, we set up an AlphaScreen homogeneous assay measuring the interaction between PoloBoxtide and full length Plk1 (see scheme in Figure 1E). The conditions were designed such that the assay could identify enhancers or inhibitors of the interactions (see Materials and Methods and Supporting Information). We then screened a collection of 1280 approved drugs and molecular probes. We 
identified that the flavonoid luteolin enhanced the interaction between PoloBoxtide and Plk1 (AC50 $20 \mu \mathrm{M}$; Figure 1F). In an overall similar approach, we established the assay measuring the interaction between PoloBoxtide and the isolated PBD (see scheme in Figure 1G). Luteolin did not affect the interaction between PoloBoxtide and the PBD (Figure 1H). A compound exposing the PBD for interaction with PoloBoxtide would be expected to release the intramolecular inhibition in Plk1 and be an "activator". However, Luteolin had been previously found to bind at the ATP-binding site of the protein kinase CK2 ${ }^{18}$. NMR-based ligand observed waterLOGSY (WL) experiment was used to investigate the interaction of the small molecule with the Plk1 kinase domain. WL is based on detecting the transfer of magnetization from the bulk solvent to the small solute (ligand) molecules. The sign of the ligand (small molecule) signal is inverted (or an increase in the intensity) when the ligand is bound to a slowly tumbling macromolecule (protein) within the sample. The presence of positive signals for the proton resonances (SI Figure 1C, signals $(*)$ ) of Luteolin in the WL spectrum acquired in the presence of both ATP-analogue and Plk1 kinase domain indicated binding to the protein. The proton signals of AMP-PNP (a nonhydrolyzable analogue of ATP) (SI Figure 1C, signals marked 2\#) displayed negative intensities and were comparable to the intensities observed in the absence of protein (SI Figure 1A, signals marked 2\#), suggesting that AMP-PNP did not bind in the presence of luteolin. These results confirmed that Luteolin binds to the Plk1 kinase domain and competes out AMP-PNP from its binding site. Luteolin inhibited the activity of Plk1 towards a polypeptide substrate which does not require a PBD docking interaction (IC50 $5.8 \mu \mathrm{M}$; Figure 1I). This is in agreement with luteolin binding at the ATP-binding site of Plk1, as described to bind to CK2 ${ }^{18}$. Interestingly, together, the above results suggested that the binding at the ATP-binding site produced allosteric effects that enhanced the binding of the PBD to PoloBoxtide. The above data provided experimental evidence of the rich allosteric communication between the PBD and the catalytic domain, which could be modulated by small compounds.

To identify new allosteric modulators of Plk1, we then screened a library comprising 14400 small compounds (average size $320 \mathrm{Da}$ ) for their ability to affect the interaction between PoloBoxtide and full-length Plk1. We identified a number of small molecules which inhibited or increased to different degrees the interaction of Plk1 with PoloBoxtide. Here, we present compounds AW00551, SCR01010 and FM00204 (Figure 2A), that exhibited two different allosteric effects. While FM00204 enhanced the interaction between full-length Plk1 and PoloBoxtide (Figure 2B; 
AC50 $=10 \mu \mathrm{M})$, SCR01010 and AW00551 weakened the interaction between PoloBoxtide and full-length Plk1 (Figure 2B; IC50=20 $\mu \mathrm{M}$ and $1 \mu \mathrm{M}$, respectively). We tested the effect of the compounds on the interaction between PoloBoxtide and the isolated PBD of Plk1. None of the compounds effected the interaction between the PBD and PoloBoxtide (Figure 2C), indicating that their effects were not by direct competition with PoloBoxtide-. Further, using the WL experiment we probed the binding of AW00551, SCR01010 and FM00204 to the isolated Plk1 kinase domain. SCR01010 was insoluble under the NMR experimental buffer conditions. WL spectra of FM00204 and AW00551 in the presence of AMP-PNP and Plk1 kinase domain showed positive signals (SI Figure 2C and 2D, signals $(*)$ ) for the compounds and negative for AMP-PNP (SI Figure 2C and 2D, signals marked 2\#). In addition, we tested the possible binding of FM00204 and AW00551 to the isolated PBD (SI Figure 3A). FM00204 did not bind to the PBD. In contrast, a waterLOGSY NMR experiment clearly detected an interaction between AW00551 and the isolated PBD of Plk1 (SI Figure 3B). Thus, we suggest that AW00551 interacts with both, the catalytic domain and the PBD (SI Figure 4C).

Further, the compounds partially inhibited the enzymatic activity of full-length (FL) and of the catalytic domain (CD) of Plk1 (Figure 2D), although the potency for the inhibition of Plk1 activity in vitro was very low. Such low potency in vitro is not expected to inhibit the cellular kinase activity significantly by interaction with the ATP-binding site of the kinase stabilized in an active conformation in the presence of at least ten times higher concentration of ATP. However, the compounds could inhibit Plk1 downstream signaling in cells by allosteric mechanisms, for example, by affecting the ability of the PBD to interact with cellular partners at precise cellular complexes.

To further describe the mechanism by which the small compounds affected the binding of Plk1 to PoloBoxtide, we next investigated the effect of the compounds on the temperature stability of Plk1 and on the isolated catalytic domain of Plk1. As a control, we tested the effect of BI6727 on the temperature stability of Plk1. Interestingly, we found that the ATP-competitive inhibitor BI6727 strongly stabilized the catalytic domain of Plk1 $\left(\Delta \mathrm{Tm}=20.8 \pm 0.02{ }^{\circ} \mathrm{C}\right.$; Figure $\left.2 \mathrm{E}\right)$ but was overall destabilizing the full-length protein $\left(\Delta \mathrm{Tm}=-11.77 \pm 0.95{ }^{\circ} \mathrm{C}\right.$; Figure $\left.2 \mathrm{~F}\right)$. Indeed, in the presence of BI6727 the graphical representation of denaturation of full-length Plk1 by temperature revealed a complex denaturation pattern, with two distinct denaturation curves. A 
FM00204 did not significantly affect the overall stability of full-length Plk1 and only mildly stabilized the catalytic domain $\left(\Delta \operatorname{Tm} 1.8 \pm 0.25^{\circ} \mathrm{C}\right)$ suggesting that it also binds to the catalytic domain of Plk1 and that, in full-length Plk1, by displacing the PBD interaction with the catalytic domain (see scheme in Figure 2H), it produces a mix of stabilization of the catalytic domain and de-stabilization of the full-length protein, which are substracted. AW00551, on the other hand, stabilized the full length Plk1 protein $\left(\Delta \mathrm{Tm} 4.98 \pm 0.7^{\circ} \mathrm{C}\right)$ whereas it also stabilized the catalytic domain $\left(\Delta \operatorname{Tm} 2.51 \pm 0.09{ }^{\circ} \mathrm{C}\right)$. Such behavior is consistent with AW00551 binding at least partially to the catalytic domain and stabilizing the inhibited form of Plk1, with the PBD attached to the catalytic domain, inhibiting the binding to PoloBoxtide (see scheme in Figure $2 \mathrm{H}$ ). SCR01010 could also have such mechanism of action since it inhibits the interaction with PoloBoxtide; however, we could not evaluate the effect of SCR01010 on the Plk1 stability assays because the compound precipitated under the conditions of the assay. The above characterizations showed that the conformation of Plk1 can be modulated by allosteric compounds, i.e. binding of the compounds to the catalytic domain can enhance or inhibit the interactions of the PBD with its phosphorylated binding partners, including substrates.

In order to gather additional information on the molecular mechanism of action of the three compounds we checked their ability to inhibit the in vitro the kinase activity of a panel of 50 protein kinases (Table S1). At $20 \mu \mathrm{M}$, FM00204 inhibited more then $50 \%$ the activity of 7 out of 
50 protein kinases in the panel. Since NMR data showed that FM00204 competes out an ATPanalogue, we can suggest that FM00204 binds at the ATP-binding site (Figure 3B). The poor selectivity of FM00204 is in agreement with FM00204 binding at the ATP-binding site in the active conformation of the kinase, and partially inhibiting different kinases with low potency (Figure 3B). In comparison, SCR01010 inhibited one kinase in the panel and AW00551 only inhibited Plk1. The NMR indicated that AW00551 binds both to the isolated catalytic domain and to the isolated PBD of Plk1; in addition, the NMR data indicated that the binding of AW00551 competed out the ATP-analogue. Therefore we suggest that AW00551 may bind to the catalytic domain and the PBD in a manner that allosterically communicates with the ATP-binding site, stabilizing the inactive structure of the ATP-binding site (option 1; Figure 3C). Since the crystal structure of the zebrafish Plk1 shows the PBD in direct proximity to the inactive structure of the ATP-binding site ${ }^{7}$, the compound could also bind at such site (option 2, Figure 3C). Since NMR experiments indicated that the binding of all compounds competed out ATP, the mechanism of inhibition is expected to be due to the lack of ATP binding and not to a modification of the $\mathrm{Km}$ for ATP. We also tested if the compounds would excert their effects on a Plk1 protein that is mutated at the ATP-binding site. Mutations at the ATP-binding site could affect the overall conformation of the kinase. Therefore, for this assay, we employed Plk1 [Cys67Val; Leu130Gly], a construct that has two mutations at the ATP-binding site and was shown to conserve its physiological functions, but has modified sensitivity to compounds binding at the ATP-binding site $^{19}$. GFP-Plk1 wt bound to biotin-PoloBoxtide and the interaction was greatly enhanced by BI6727 and FM00204 while the interaction was robustly inhibited by AW00551 and SCR01010. GFP-Plk1 [Cys67Val; Leu130Gly] also bound biotin-PoloBoxtide, however, the interaction was not affected by BI6727 or FM00204 (SI Figure 4). The finding confirmed that FM00204, like BI6727, indeed bind to the ATP-binding site. However, AW00551 and SCR01010 also inhibited to some degree the interaction of biotin-PoloBoxtide with GFP-Plk1 [Cys67Val; Leu130Gly]. Therefore the binding site of AW00551 and SCR01010 remained undefined between option 1 and option 2 shown in Figure 3.

In previous work we showed how the interaction of substrates at a docking site on the protein kinase PDK1 can regulate the activity of the catalytic domain by allosterically affecting the conformation of the ATP-binding site ${ }^{20-22}$. Interestingly, we also described compounds binding at the ATP-binding site on PDK1, which can modulate the conformation of the docking site, by the 


\section{FM00204 and SCR01010 influence the interaction of Plk1 with Bora and the phosphorylation of substrates in cells}

Hit compounds identified in an in vitro screening using pure components may be inactive or nonspecific in cells. Luteolin was potently inhibiting cellular proliferation but we could not identify a primarily effect on Plk1. In contrast, we identified that FM00204 and SCR01010 selectively affected Plk1 signalling and were characterized in depth. Plk1 is activated by Aurora A, which phosphorylates Plk1 at T210 in $\mathrm{G}_{2}$. This phosphorylation event requires the presence of the cofactor Bora 27, 28. To examine the effect of compounds on a specific Plk1 protein-protein interaction, we synchronized cells in mitosis by Nocodazole treatment and probed the effects on the Plk1/Bora complex (Figure 4A). To study the Plk1/Bora interaction, we immunoprecipitated Plk1 and analyzed the amount of Bora that co-immunoprecipitated. Interestingly, the Plk1/Bora interaction was blocked by SCR01010 and augmented by FM00204 (Figure 4B) supporting the model that the allosteric compounds SCR01010 and FM00204 influenced the three-dimensional structure of Plk1 and modulated the ability of Plk1 to form complexes in a cellular setting.

Next, we studied the effect of compounds on specific Plk1 substrates (TCTP, Myt1, the cdc2-activating phosphatase $\mathrm{Cdc} 25 \mathrm{c}$ ) in compound-treated cells. The translationally controlled tumor protein TCTP was identified as a protein that binds to the PBD of Plk1 ${ }^{29}$. Subsequently, 
Plk1 phosphorylates TCTP on two serine residues. In addition, Plk1 phosphorylates Myt1, an inhibitory kinase for the MPF, and the phosphatase Cdc25c during M phase ${ }^{30,31}$. Since FM00204 was shown to enhance the interaction between full-length Plk1 and PoloBoxtide (Figure 4B), we analyzed the phosphorylation of TCTP, Cdc25c and Myt1 by Plk1 using phospho-specific antibodies. Interestingly, in the presence of FM00204 we observed an increase of Plk1-specific phosphorylation of all three proteins (Figure 4C). Remarkably, the phospho-peptide, PoloBoxtide, used for the assays depicted in figure 2 represents the binding site for Plk1 in Cdc25c. We could demonstrate that FM00204 promotes not only the binding of PoloBoxtide to full-length Plk1, but also the phosphorylation of Cdc25c which might suggest improved binding of Cdc25c and Plk1 in cells.

In contrast, in cells treated with SCR01010, that reduces the binding of PoloBoxtide to fulllength Plk1 (Fig. 2B), a decreased phosphorylation of Myt1 was observed (Figure 4C). The phosphorylation of TCTP and Cdc25C remained below the limit of detection. Although both compounds, SCR01010 and FM00204, had a weak inhibitory effect on Plk1 catalytic activity in vitro, the augmented binding of the substrate proteins TCTP, Myt1 and Cdc25c seemed to be sufficient for their enhanced phosphorylation by Plk1.

\section{FM00204 and SCR01010 inhibit cell proliferation by arresting cells in $\mathrm{G}_{2} / \mathrm{M}$ and promoting apoptosis}

Owing to the essential role of Plk1 in mitosis ${ }^{32,33}$ it is expected that interfering with its function using small molecule inhibitors would generate a mitotic arrest accompanied by an increase in mitotic index. Measurements by flow cytometry revealed that the treatment of HeLa cells with SCR01010 and FM00204 increased the proportion of mitotic cells (SI Figure 5A). While SCR01010 $(25 \mu \mathrm{M})$ induced a mitotic arrest of 34\%, the treatment with $25 \mu \mathrm{M}$ FM00204 arrested $80 \%$ of the cells in $\mathrm{G}_{2} / \mathrm{M}$ (SI Figure $5 \mathrm{~A}$ ). The induction of a mitotic arrest by both compounds was confirmed in western blot analyses demonstrating an increase of hyperphosphorylation of Cdc25c, a characteristic feature of mitotic cells (SI Figure 5B). Furthermore, we observed an increase in the levels of the mitotic markers Plk1, Cyclin B1 and phosphohistone H3 (SI Figure 5B). SCR01010 and FM00204 inhibited the proliferation of HeLa cells in a dose-dependent manner (SI Figure 5C). Cancer cells with inhibition of Plk1 undergo apoptosis, mostly caused by 
mitotic catastrophe. To corroborate that inhibiting Plk1 function triggers cancer cells to apoptosis, asynchronous HeLa cells were treated with increasing amounts of both compounds for 24 hours. Indeed, we found a dose-dependent increase in apoptosis induction by annexin staining (SI Figure 5D).

The inhibition of Plk1 in cells can be followed by studying the phenotype of treated cells using immunofluorescence microscopy (Figure 5A). The phenotypes induced by FM00204 and SCR01010 were compared to those generated by the ATP-competitive inhibitor BI6727 ${ }^{5}$ and Poloxin ${ }^{9}$. FM00204 (20 $\left.\mu \mathrm{M}\right)$ and SCR01010 (20 $\left.\mu \mathrm{M}\right)$ caused a prometaphase arrest associated with a mitotic index of $58 \%$ and $42 \%$, respectively compared to $14 \%$ for Poloxin $(20 \mu \mathrm{M})$ and 59\% for BI6727 (100 nM) (Figure 5A,B). SCR01010 and FM00204 displayed different effects on mitotic spindles. SCR01010 generated mainly aberrant, thin and small spindles (57\%), which were to some degree comparable to a Poloxin-induced phenotype (Figure $5 \mathrm{~A}, \mathrm{C}$ ). On the other hand, FM00204 showed an accumulation of multipolar spindles (45\%) (Figure 5 AD). Moreover, we could confirm the findings of several studies reporting that BI6727, at a concentration of 100 $\mathrm{nM}$, generates exclusively monopolar spindles (Figure $5 \mathrm{~A}, \mathrm{E}$ ). The different phenotypes generated by SCR01010 and FM00204 suggest different mechanisms of action of Plk1 inhibition implicated in spindle nucleation and centrosome maturation or separation during early mitotic phases ${ }^{34-36}$. The effects of SCR01010 and FM00204 on chromosome dynamics were also examined by immunofluorescence. Similar to Poloxin or to BI6727, both compounds SCR01010 and FM00204 generated a high rate of chromosome congression failure following $16 \mathrm{~h}$ after the treatment, up to $73 \%, 100 \%, 78 \%$ and $58 \%$ respectively for Poloxin, BI6727, SCR01010 and FM00204 (Figure 4 A,F).

\section{Plk1 allosteric modulators affect the cellular localization of Plk1}

The PBD of Plk1 is known to be required for Plk1 localization at centrosomes and kinetochores during mitosis. The PBD is proposed to mediate this task by docking to phosphorylated sites generated by other priming kinases ${ }^{8,37}$. However, it has also been reported that the kinase activity is also involved in Plk1's mitotic sub-localizations by generating its own phosphosites for PBD binding (self-priming) ${ }^{38,39}$. We therefore sought to investigate whether SCR01010 and FM00204 affect the localization of Plk1 during early phases of mitosis. Thus, we stained HeLa cells with $\gamma$-Tub and with the anti-centromere antibody ACA as centrosome and centromere 
marker. Whereas control mitotic HeLa cells showed a clear centrosome and kinetochore localization of Plk1, all other treatments led to its partial removal from centrosome and kinetochores. The strongest mislocalization of Plk1 from centrosomes and kinetochores was achieved after treating cells with SCR01010 (42\%). The mislocalization induced by treatment with Poloxin (32\%) or FM00204 (31\%) was less pronounced (Figure 5 G,H). Eventhough a high number of BI6727-treated cells showed the presence of Plk1 at centrosome and kinetochore, this signal was very weak and faint compared to control cells (Figure 5 F,H). Intriguingly, treating cells with SCR01010 or FM00204 produced large Plk1 aggregates, that were seen either in proximity to the DNA or even outside the chromosomal zone, which indicates that these large foci were unlikely the result of abnormal kinetochore recruitment of Plk1 (Figure 5G, white arrow heads). A similar observation of large Plk1 aggregates was made when cells were treated with Poloxin or BI6727 but only to a lesser extent (Figure 5G). These data confirm that SCR01010 and FM00204 trigger similar phenotypes as those generated by the established Plk1 inhibitors (Poloxin and BI6727) and strongly argue for the specificity of these two new compounds toward Plk1.

Together, the present work describes the allosteric modulation of Plk1 by small compounds. Our results suggest that the effect of inhibiting the cellular function of Plk1 may be equally observed by compounds that potently inhibit the enzymatic activity of Plk1 or by allosteric modulators that are weak inhibitors of Plk1 enzymatic activity. SCR01010 and FM00204 specifically inhibited Plk1 function in cells by affecting the conformation and mechanism of regulation of Plk1, enhancing or inhibiting the binding to phosphorylated epitopes that participate in the dynamic proper localization of Plk1. BI6727, luteolin and FM00204 open the structure of Plk1 thereby releasing the inhibition that the PBD exerts on the catalytic domain. In addition, BI6727 potently inhibits the catalytic activity of Plk1. In contrast, the mechanism of action of AW00551 and SCR01010 appear to produce a different set of effects, inhibit the binding to phosphorylated epitopes to the PBD and stabilize the PBD-catalytic domain interaction that physiologically inhibits Plk1. Such mechanism could explain how compounds with different mode of action produce different effects on cells. Interestingly, we describe here that the effect of SCR01010 and FM00204 is to disturb the proper intermolecular interactions of Plk1 and promote mislocalization of Plk1. In these cases, by mislocalizing the kinase, the inhibition of physiological function of Plk1 could last for longer periods of time after the compound has dissociated from Plk1. 


\section{Materials and Methods}

Details on the proteins used, the AlphaScreen interaction assays, the screening of libraries, the in vitro kinase activity assay, the temperature stability assay and cell-based assays are presented under Supporting Information. In brief, GST-His-Plk1 FL was from ProQinase; His-Plk1 FL and His-Plk1 CD (13-345) were produced from pTriEX1.1 vector in insect cells. His-PBD was produced in bacteria as described ${ }^{10}$. NMR methods are described in the text and in the Supplementary Information. The interaction assays were performed using the AlphaScreen technology following the general guidelines of the manufacturers (Perkin Elmer). The interaction assay contained $30 \mathrm{nM}$ Plk1 and 50-100 nM PoloBoxtide in $50 \mathrm{mM}$ Tris- $\mathrm{HCl}$ (pH 7.4), $100 \mathrm{mM}$ $\mathrm{NaCl}, 2 \mathrm{mM}$ dithiotreitol, $0.01 \%$ (v/v) Tween-20 and 0.1\% (w/v) BSA. The screened libraries (50 $\mu \mathrm{M})$ were the FDA-approved library (Prestwick) and a library comprising 14400 diverse compounds (Hiftinder, from Maybridge). The individual compounds in the library have been analyzed by the manufacturer by appropriate methodologies including NMR, FT-IR, LC-MS, 
HPLC and elemental analysis, with greater than $90 \%$ purity, with majority of compounds with more than $95 \%$ purity; reactive compounds are excluded from the Hitfinder library. Individual compounds were purchased from Maybridge. Compounds subjected to NMR studies were confirmed to have high purity and the expected chemical features. The Plk activity assay was performed in a mix containing $50 \mathrm{mM}$ Tris- $\mathrm{HCl} \mathrm{pH} 7.5,0.05 \mathrm{mg} / \mathrm{ml} \mathrm{BSA}, 0.1 \%$ (v/v) 2mercaptoethanol, $10 \mathrm{mM} \mathrm{MgCl} 2,100 \mu \mathrm{M}\left[\gamma^{32} \mathrm{P}\right] \mathrm{ATP}$ (5-50 cpm/pmol), 0.003\% Brij, 150-350 ng GST-His-Plk1, and the peptide substrate (KKGGSFNDTLDFD, $100 \mu \mathrm{M}$ ) as performed for other kinases ${ }^{22}$. Temperature stability assays were performed using the differential scanning fluorimetry as previously performed ${ }^{21}$. Cell-based assays were performed using standard protocols ${ }^{40}$. Unless specified otherwise, experiments were performed at least in triplicate. Statistics were analyzed by Student's t-test (two-sided, paired). Significant differences are indicated with an asterisk $(* \mathrm{P} \leq 0.05 ; * * \mathrm{P} \leq 0.01 ; * * * \mathrm{P} \leq 0.001)$.

Supporting Information is available, including Supplementary methods, SI Table 1 and SI Figure 1-5. This material is available free of charge via the internet at http://pubs.acs.org.

\section{Author contributions}

LP set-up all biochemical assays, performed biochemical assays and analyzed data; IB performed the screenings and analyzed data; ES and AKH performed additional biochemical assays and analyzed data; SG, KS, BC, DK, expressed and purified Plk constructs either eukaryotically or prokaryotically; SS performed the NMR experiments and analysis. HS directed the expression and purification of Plk constructs and the NMR experiments. MR and MS performed the cellular assays. KSt directed the cellular work. MR, MS and KSt analyzed the cellular data. RMB directed the molecular biology, biochemical work and analyzed data. RMB and KSt wrote the manuscript with support from HS, KS, SS, LP, AKH, MR and MS.

\section{Acknowledgements}

We thank Y. Matthess, E. Proschak, D. Odadzic, the members of the DKTK-Frankfurt network and Research Group PhosphoSites for support and discussions. The work was supported by the German Cancer Consortium (DKTK) to KSt, HS and RMB, DFG BI 1044/13-1, ANPCyT (subsidio PICT PRH-2016-3525), FOCEM-Mercosur (COF 03/11) and CONICET. 
1. Manning, G., Whyte, D. B., Martinez, R., Hunter, T., and Sudarsanam, S. (2002) The protein kinase complement of the human genome, Science 298, 1912-1934.

2. Pawson, T., and Scott, J. D. (2005) Protein phosphorylation in signaling--50 years and counting, Trends Biochem Sci 30, 286-290.

3. Huse, M., and Kuriyan, J. (2002) The conformational plasticity of protein kinases, Cell 109, 275-282.

4. Davis, M. I., Hunt, J. P., Herrgard, S., Ciceri, P., Wodicka, L. M., Pallares, G., Hocker, M., Treiber, D. K., and Zarrinkar, P. P. (2011) Comprehensive analysis of kinase inhibitor selectivity, Nat Biotechnol 29, 1046-1051.

5. Rudolph, D., Steegmaier, M., Hoffmann, M., Grauert, M., Baum, A., Quant, J., Haslinger, C., GarinChesa, P., and Adolf, G. R. (2009) BI 6727, a Polo-like kinase inhibitor with improved pharmacokinetic profile and broad antitumor activity, Clin Cancer Res 15, 3094-3102.

6. Cheng, K. Y., Lowe, E. D., Sinclair, J., Nigg, E. A., and Johnson, L. N. (2003) The crystal structure of the human polo-like kinase-1 polo box domain and its phospho-peptide complex, EMBO J 22, 57575768.

7. Xu, J., Shen, C., Wang, T., and Quan, J. (2013) Structural basis for the inhibition of Polo-like kinase 1, Nat Struct Mol Biol 20, 1047-1053.

8. Elia, A. E., Rellos, P., Haire, L. F., Chao, J. W., Ivins, F. J., Hoepker, K., Mohammad, D., Cantley, L. C., Smerdon, S. J., and Yaffe, M. B. (2003) The molecular basis for phosphodependent substrate targeting and regulation of Plks by the Polo-box domain, Cell 115, 83-95.

9. Reindl, W., Yuan, J., Kramer, A., Strebhardt, K., and Berg, T. (2008) Inhibition of polo-like kinase 1 by blocking polo-box domain-dependent protein-protein interactions, Chem Biol 15, 459-466.

10. Scharow, A., Raab, M., Saxena, K., Sreeramulu, S., Kudlinzki, D., Gande, S., Dotsch, C., KurunciCsacsko, E., Klaeger, S., Kuster, B., Schwalbe, H., Strebhardt, K., and Berg, T. (2015) Optimized PIk1 PBD Inhibitors Based on Poloxin Induce Mitotic Arrest and Apoptosis in Tumor Cells, Acs Chem Biol 10, 2570-2579.

11. Qin, T., Chen, F., Zhuo, X., Guo, X., Yun, T., Liu, Y., Zhang, C., and Lai, L. (2016) Discovery of Novel Polo-Like Kinase 1 Polo-Box Domain Inhibitors to Induce Mitotic Arrest in Tumor Cells, $J$ Med Chem 59, 7089-7096.

12. Lee, K. S., Burke, T. R., Jr., Park, J. E., Bang, J. K., and Lee, E. (2015) Recent Advances and New Strategies in Targeting Plk1 for Anticancer Therapy, Trends Pharmacol Sci 36, 858-877.

13. Fang, Z., Grutter, C., and Rauh, D. (2013) Strategies for the selective regulation of kinases with allosteric modulators: exploiting exclusive structural features, Acs Chem Biol 8, 58-70.

14. Fabbro, D. (2015) 25 years of small molecular weight kinase inhibitors: potentials and limitations, $\mathrm{Mol}$ Pharmacol 87, 766-775.

15. Changeux, J. P. (2013) 50 years of allosteric interactions: the twists and turns of the models, Nat Rev Mol Cell Biol 14, 819-829.

16. Nussinov, R., and Tsai, C. J. (2013) Allostery in disease and in drug discovery, Cell 153, 293-305.

17. Lansing, T. J., McConnell, R. T., Duckett, D. R., Spehar, G. M., Knick, V. B., Hassler, D. F., Noro, N., Furuta, M., Emmitte, K. A., Gilmer, T. M., Mook, R. A., Jr., and Cheung, M. (2007) In vitro biological activity of a novel small-molecule inhibitor of polo-like kinase 1, Mol Cancer Ther 6, 450-459.

18. Lolli, G., Cozza, G., Mazzorana, M., Tibaldi, E., Cesaro, L., Donella-Deana, A., Meggio, F., Venerando, A., Franchin, C., Sarno, S., Battistutta, R., and Pinna, L. A. (2012) Inhibition of protein kinase CK2 by flavonoids and tyrphostins. A structural insight, Biochemistry 51, 6097-6107. 
19. Burkard, M. E., Randall, C. L., Larochelle, S., Zhang, C., Shokat, K. M., Fisher, R. P., and Jallepalli, P. V. (2007) Chemical genetics reveals the requirement for Polo-like kinase 1 activity in positioning RhoA and triggering cytokinesis in human cells, Proc Natl Acad Sci U S A 104, 4383-4388.

20. Biondi, R. M., Cheung, P. C., Casamayor, A., Deak, M., Currie, R. A., and Alessi, D. R. (2000) Identification of a pocket in the PDK1 kinase domain that interacts with PIF and the C-terminal residues of PKA, Embo J 19, 979-988.

21. Busschots, K., Lopez-Garcia, L. A., Lammi, C., Stroba, A., Zeuzem, S., Piiper, A., Alzari, P. M., Neimanis, S., Arencibia, J. M., Engel, M., Schulze, J. O., and Biondi, R. M. (2012) Substrate-Selective Inhibition of Protein Kinase PDK1 by Small Compounds that Bind to the PIF-Pocket Allosteric Docking Site, Chem Biol 19, 1152-1163.

22. Engel, M., Hindie, V., Lopez-Garcia, L. A., Stroba, A., Schaeffer, F., Adrian, I., Imig, J., Idrissova, L., Nastainczyk, W., Zeuzem, S., Alzari, P. M., Hartmann, R. W., Piiper, A., and Biondi, R. M. (2006) Allosteric activation of the protein kinase PDK1 with low molecular weight compounds, Embo J 25, 5469-5480.

23. Schulze, J. O., Saladino, G., Busschots, K., Neimanis, S., Suss, E., Odadzic, D., Zeuzem, S., Hindie, V., Herbrand, A. K., Lisa, M. N., Alzari, P. M., Gervasio, F. L., and Biondi, R. M. (2016) Bidirectional Allosteric Communication between the ATP-Binding Site and the Regulatory PIF Pocket in PDK1 Protein Kinase, Cell Chem Biol 23, 1193-1205.

24. Wang, L., Perera, B. G., Hari, S. B., Bhhatarai, B., Backes, B. J., Seeliger, M. A., Schurer, S. C., Oakes, S. A., Papa, F. R., and Maly, D. J. (2012) Divergent allosteric control of the IRE1alpha endoribonuclease using kinase inhibitors, Nat Chem Biol 8, 982-989.

25. Hatzivassiliou, G., Song, K., Yen, I., Brandhuber, B. J., Anderson, D. J., Alvarado, R., Ludlam, M. J., Stokoe, D., Gloor, S. L., Vigers, G., Morales, T., Aliagas, I., Liu, B., Sideris, S., Hoeflich, K. P., Jaiswal, B. S., Seshagiri, S., Koeppen, H., Belvin, M., Friedman, L. S., and Malek, S. (2010) RAF inhibitors prime wild-type RAF to activate the MAPK pathway and enhance growth, Nature 464, 431-435.

26. Poulikakos, P. I., Zhang, C., Bollag, G., Shokat, K. M., and Rosen, N. (2010) RAF inhibitors transactivate RAF dimers and ERK signalling in cells with wild-type BRAF, Nature 464, 427-430.

27. Seki, A., Coppinger, J. A., Jang, C. Y., Yates, J. R., and Fang, G. (2008) Bora and the kinase Aurora a cooperatively activate the kinase Plk1 and control mitotic entry, Science 320, 1655-1658.

28. Macurek, L., Lindqvist, A., Lim, D., Lampson, M. A., Klompmaker, R., Freire, R., Clouin, C., Taylor, S. S., Yaffe, M. B., and Medema, R. H. (2008) Polo-like kinase-1 is activated by aurora A to promote checkpoint recovery, Nature 455, 119-123.

29. Yarm, F. R. (2002) Plk phosphorylation regulates the microtubule-stabilizing protein TCTP, Mol Cell Biol 22, 6209-6221.

30. Nakajima, H., Toyoshima-Morimoto, F., Taniguchi, E., and Nishida, E. (2003) Identification of a consensus motif for Plk (Polo-like kinase) phosphorylation reveals Myt1 as a Plk1 substrate, J Biol Chem 278, 25277-25280.

31. Toyoshima-Morimoto, F., Taniguchi, E., and Nishida, E. (2002) Plk1 promotes nuclear translocation of human Cdc25C during prophase, EMBO Rep 3, 341-348.

32. Strebhardt, K. (2010) Multifaceted polo-like kinases: drug targets and antitargets for cancer therapy, Nat Rev Drug Discov 9, 643-660.

33. Strebhardt, K., and Ullrich, A. (2006) Targeting polo-like kinase 1 for cancer therapy, Nat Rev Cancer 6, 321-330.

34. Lane, H. A., and Nigg, E. A. (1996) Antibody microinjection reveals an essential role for human pololike kinase 1 (Plk1) in the functional maturation of mitotic centrosomes, J Cell Biol 135, 17011713. 
35. Sumara, I., Gimenez-Abian, J. F., Gerlich, D., Hirota, T., Kraft, C., de la Torre, C., Ellenberg, J., and Peters, J. M. (2004) Roles of polo-like kinase 1 in the assembly of functional mitotic spindles, Curr Biol 14, 1712-1722.

36. van Vugt, M. A., van de Weerdt, B. C., Vader, G., Janssen, H., Calafat, J., Klompmaker, R., Wolthuis, R. M., and Medema, R. H. (2004) Polo-like kinase-1 is required for bipolar spindle formation but is dispensable for anaphase promoting complex/Cdc20 activation and initiation of cytokinesis, $J$ Biol Chem 279, 36841-36854.

37. Hanisch, A., Wehner, A., Nigg, E. A., and Sillje, H. H. (2006) Different Plk1 functions show distinct dependencies on Polo-Box domain-mediated targeting, Mol Biol Cell 17, 448-459.

38. Kang, Y. H., Park, J. E., Yu, L. R., Soung, N. K., Yun, S. M., Bang, J. K., Seong, Y. S., Yu, H., Garfield, S., Veenstra, T. D., and Lee, K. S. (2006) Self-regulated Plk1 recruitment to kinetochores by the Plk1PBIP1 interaction is critical for proper chromosome segregation, Mol Cell 24, 409-422.

39. Neef, R., Preisinger, C., Sutcliffe, J., Kopajtich, R., Nigg, E. A., Mayer, T. U., and Barr, F. A. (2003) Phosphorylation of mitotic kinesin-like protein 2 by polo-like kinase 1 is required for cytokinesis, J Cell Biol 162, 863-875.

40. Helmke, C., Raab, M., Rodel, F., Matthess, Y., Oellerich, T., Mandal, R., Sanhaji, M., Urlaub, H., Rodel, C., Becker, S., and Strebhardt, K. (2016) Ligand stimulation of CD95 induces activation of Plk3 followed by phosphorylation of caspase-8, Cell Res 26, 914-934. 


\section{Figure legends}

Figure 1. Mechanism of regulation of Plk1 by interaction of phosphopeptide with the PBD domain and screening for compounds that modulate the interaction. (A) Representation of the linear structure of Plk1, consisting of an $\mathrm{N}$-terminal protein kinase catalytic domain and a $\mathrm{C}$ terminal PBD domain. (B) Representation of the inhibited form of Plk1. The PBD domain folds onto the catalytic domain, inhibiting the catalytic activity. (C) Representation of the activated form of the kinase. The phosphopeptide PoloBoxtide binds to the PBD domain and releases the intramolecular interaction that inhibits the kinase. (D) PoloBoxtide activates Plk1 in vitro. (E) interaction between full length Plk1 and PoloBoxtide using the alphascreen technology. Upon excitation, the donor beads -D- produces oxygen singlets; if the two partners interact, the oxygen singlet excites the acceptor bead $-\mathrm{A}$ - that produces light. This homogeneous assay was used to screen for compounds that modulate the interaction. (F) Interaction between PoloBoxtide and the isolated PBD domain of Plk1 using AlphaScreen. (G) Luteolin, enhances the interaction between Plk1-FL and PoloBoxtide in vitro. (H) Luteolin does not affect the interaction between PoloBoxtide and the isolated PBD domain. (I) Luteolin inhibited the catalytic activity of Plk1, presumably by binding to the ATP-binding site and directly competing for the binding of ATP. Values represent the mean \pm the standard deviation (SD).

Figure 2. In vitro characterization of small compounds that modulate the conformation of Plk1, enhancing or inhibiting the interaction with PoloBoxtide. (A) Chemical structures of compounds identified in the screening of a library of a diverse set of small compounds. (B) Effect of small compounds on the interaction between Plk1 and PoloBoxtide. (C) Effect of small compounds on the interaction between PoloBoxtide and the isolated PBD domain of Plk1. (D) Effect of small compounds on the in vitro kinase activity of Plk1 full-length (FL) and on the isolated catalytic domain (CD) of Plk1. (E) Denaturation of Plk1 CD by temperature and stabilization by BI6727. (F) Denaturation of Plk1 FL by temperature and the effect of BI6727 on the stability. (G) BI6727 enhances the interaction between Plk1 FL and PoloBoxtide. (H) Schematic representation of the mechanism of action of small compounds that modulate the exposure of the PBD. Left, BI6727 binds to the ATP-binding site on the catalytic domain and potently inhibits the catalytic activity; in addition, our results indicate that BI6727 releases the intramolecular interaction with the PBD 
Figure 3. Schematic model on the proposed allosteric communication between the polo-box domain and the catalytic domain in Plk1 by BI6727, FM00204 and SCR01010/AW00551. (A) BI6727 binds to the ATP-binding site on the catalytic domain of Plk1, opens the Plk1 structure, enhancing interaction of PBD with phosphorylation-dependent interactions, stabilizing interactions with substrates and PBD-dependent molecular complexes, possibly affecting the proper dynamic localization of Plk1. In the right side of the panel, ATP is shown in red. Besides stabilizing the "open-active" structure, BI6727 is a high affinity inhibitor and therefore displaces ATP from the ATP-binding site. In this manner, BI6727 affects the dynamic proper location of Plk1 and also inhibits the cellular phosphorylation of all substrates. (B) FM00204 binds to the catalytic domain of Plk1 producing an enhancement of the interaction between the PBD and phosphopeptide substrates. The binding of FM00204 displaces the binding of a probe to the ATPbinding site and does not affect a Plk1 protein mutated at the ATP-binding site. Therefore, we conclude that FM00204 binds at the ATP-binding site in the active conformation, like BI6727, Our study suggests that FM00204 inhibits Plk1 cellular functions mainly by affecting its proper dynamic location, enhancing the PBD interaction with phosphoproteins. FM00204 does not bind with high affinity. Therefore, in the right section of the panel we propose that upon the release of FM00204, Plk1 would have the ability to bind ATP and phosphorylate the PBD-mediated colocalized substrates. (C) AW00551 binds to the catalytic domain of Plk1 and to the PBD of Plk1, inhibits the interaction of the PBD with phosphopeptides, stabilizing the close-inactive structure. In addition, the binding of AW00551 to the catalytic domain of Plk1 competes out the probe at the ATP-binding site. Based on the data obtained, we suggest that AW00551 makes interactions with both the catalytic domain and the PBD, stabilizing the closed-inactive structure of Plk1, where the PBD interacts with the catalytic domain and the ATP is in an inactive conformation. In the closed-inactive conformation, the PBD occludes part of the ATP-binding site. Therefore, the binding site of AW00551 could be at an interface between the catalytic domain and the PBD shown in option 1 or alternatively AW00551 could also bind at the inactive structure of the ATP- 
binding site as depicted in the cartoon shown in option 2. It is proposed that once Plk1 has lost its proper dynamic location in the cell, the release of an inhibitor with such mechanism of action (i.e. SCR01010) would not enable Plk1 to regain its physiological activity towards substrates (right section of the panel).

Figure 4. Impact of SCR01010 and FM00204 on Plk1 and its interacting proteins in cells. (A) The representative quantification of the cell cycle analysis by FACS showing cells treated for 24 $\mathrm{h}$ with compounds. (B) (left panel) Cells were treated followed by immunoblotting of the lysates for Plk1, Bora, Cyclin B1, and $\beta$-Actin. (right panel) Immunoprecipitation of Plk1 from asynchronous, SCR01010 and FM00204-treated cells. Plk1-interacting proteins were analyzed using western blot for Plk1, pT210 and Bora. (C) Cells were treated followed by immunoblotting of the lysates for pMyt1, phosho-Cdc25C (pcdc25C), Cdc25C, pTCTP, TCTP,bPlk1, Cyclin B1, phosho-Histone $\mathrm{H} 3$ (pH3), and $\beta$-Actin.

Figure 5. The allosteric inhibitors FM00204 and SCR01010 interfere with Plk1's subcellular localization in mitosis, generate aberrant and multipolar spindles, as well as an increase in chromosomal congression failure. HeLa cell were treated overnight with $25 \mu$ M Poloxin, $100 \mathrm{nM}$ BI6727, $25 \mu \mathrm{M}$ SCR01010 and 25 $\mu \mathrm{M}$ FM00240. Cells were fixed and prepared for immunofluorescence. (A) In order to assess the spindle phenotypes induced by the allosteric Plk1 inhibitors, HeLa cells were fixed and stained for Plk1, $\alpha$-tubulin, ACA (anti-centromere antibodies) and DNA. Examples of inhibitor dependent spindle phenotypes are shown (scale bar= $10 \mu \mathrm{m}$ ). (B) The mitotic indices within the different treatment groups were scored by microscopy using DAPI staining. The results are presented as means \pm SD ( $n=400-600$ cells). (C) Frequencies of cells with abnormal spindle formation. The results are represented as means \pm SD ( $\mathrm{n}=300-400$ cells). (D) The proportion of cells showing multipolar spindle. The results are represented as means $\pm \mathrm{SD}(\mathrm{n}=300-400$ cells $)$. (E) The rate of cells presenting monopolar spindles in the different treatment groups. The results are represented as means \pm SD $(n=300$ 400 cells). (F) The percentages of chromosome congression failure were defined microscopically using DAPI and represented graphically. The results are represented as means \pm SD ( $\mathrm{n}=300-400$ cells). (G) Examples of cells displaying Plk1 displacement from centrosome/kinetochore upon treatment with the inhibitors. $\delta$-tubulin and ACA were considered as marker for centrosome and 
kinetochore respectively (scale bar= $10 \mu \mathrm{M}$ ). (H) Quantification of Plk1 displacement from centrosome/kinetochore upon treatment with the allosteric inhibitors. The results are showed as means \pm SD $(n=300-400$ cells $)$. 
Figure 1

A

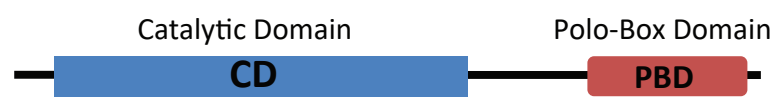

D

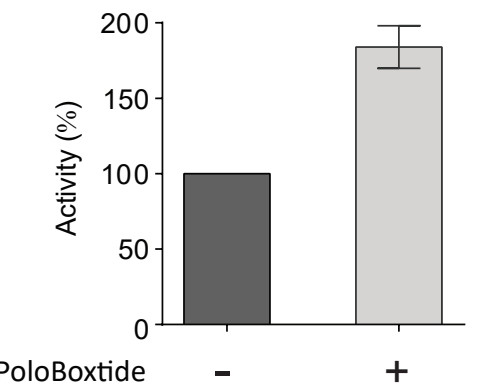

G

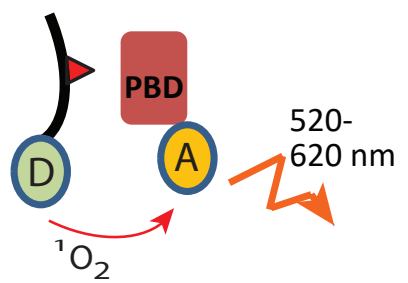

$\mathrm{H}$
B

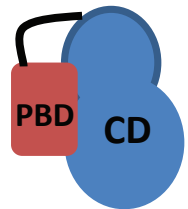

Inactive
C

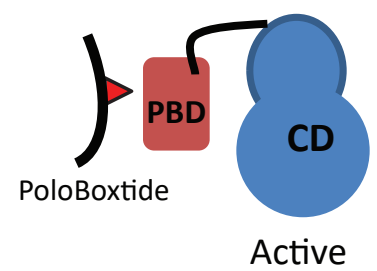

F
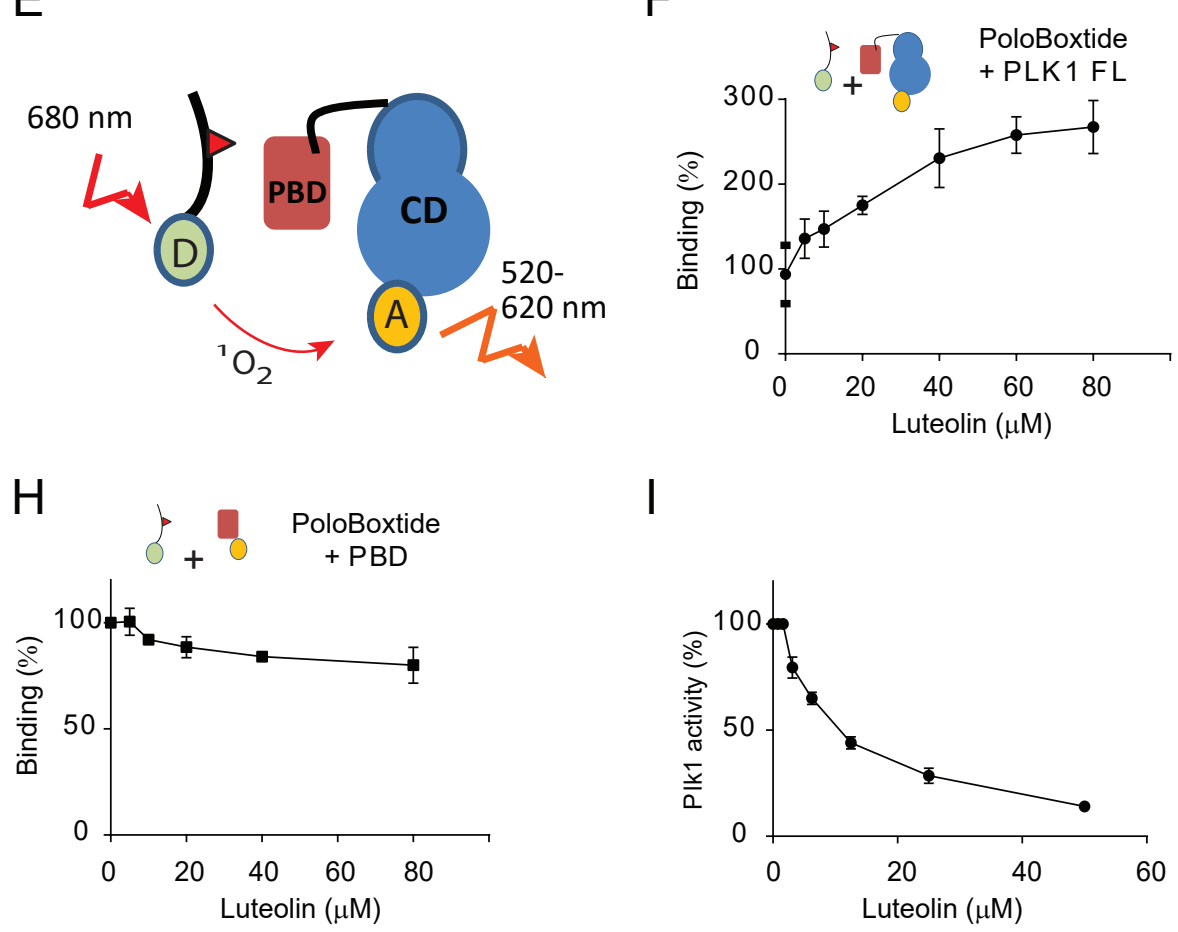
Figure 2

A

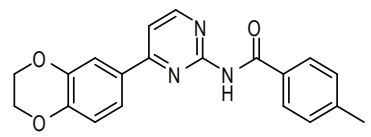

FM00204
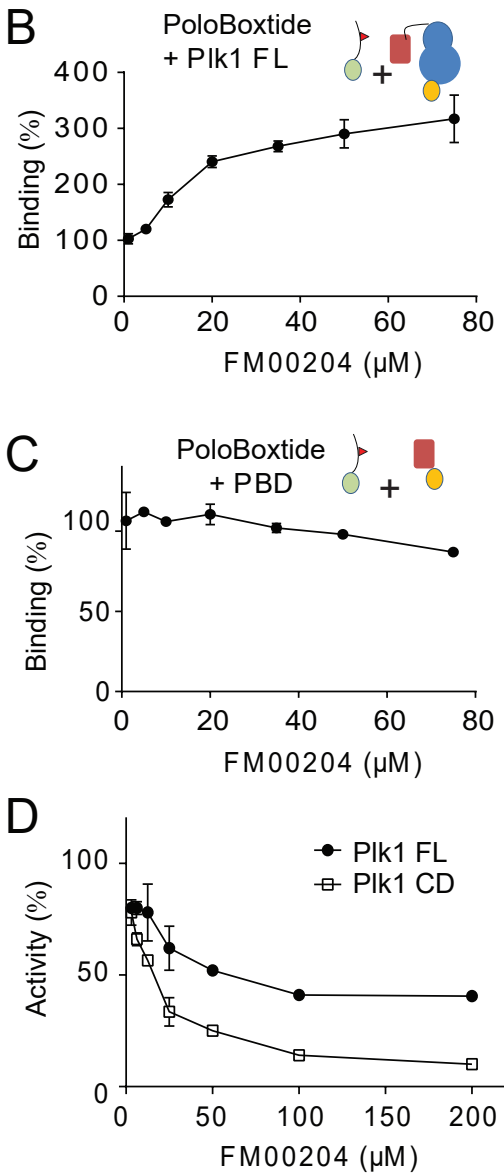

E

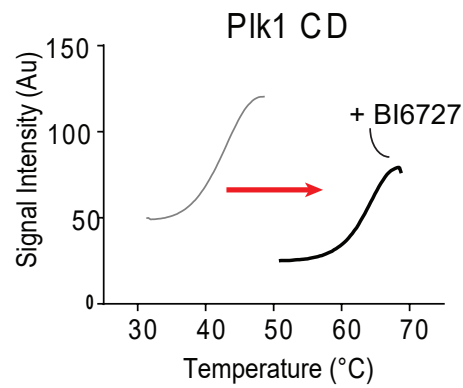

$\mathrm{H}$

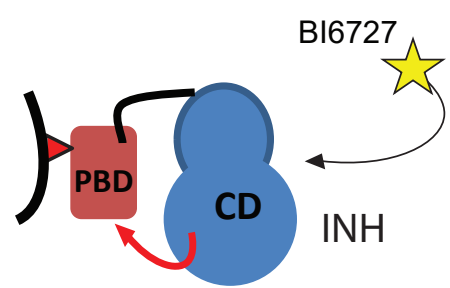

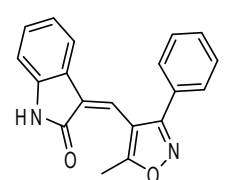

SCR01010
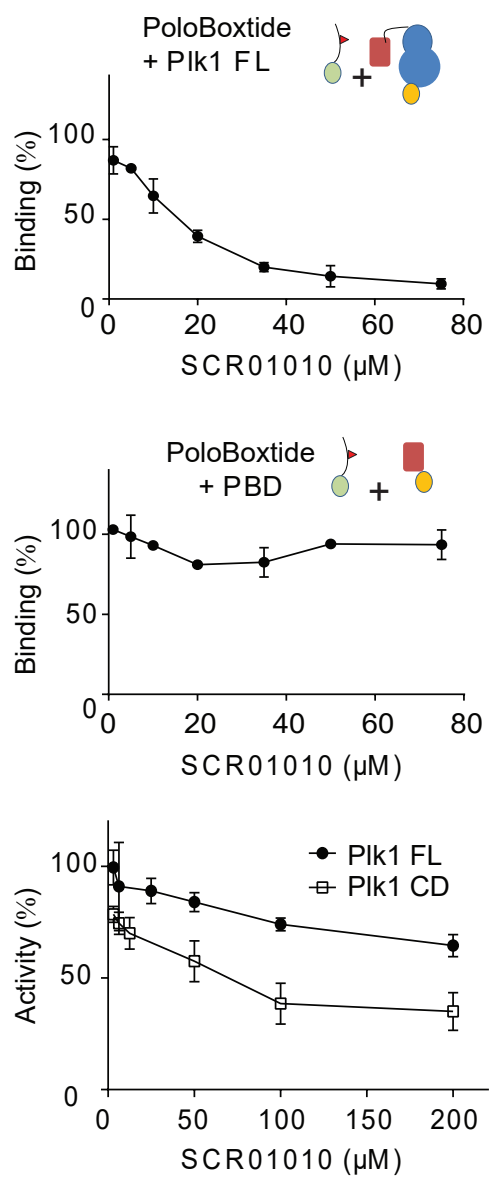

F

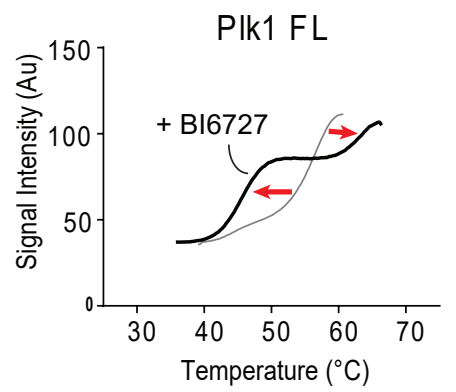

FM00204

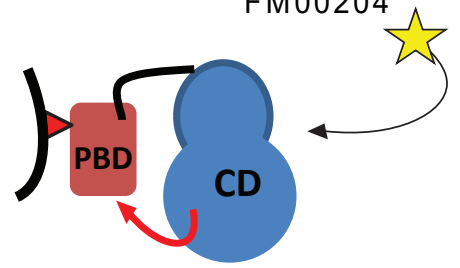

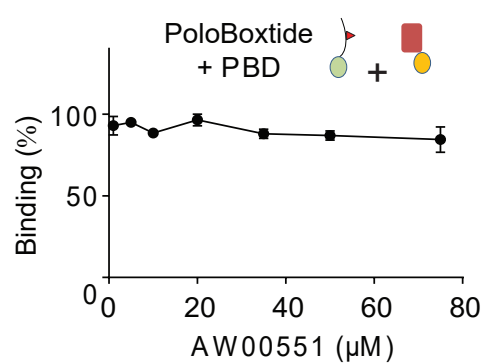

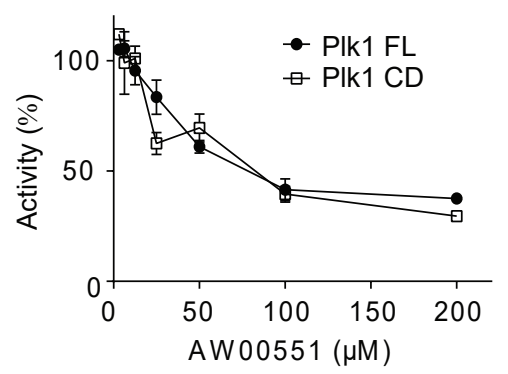

G

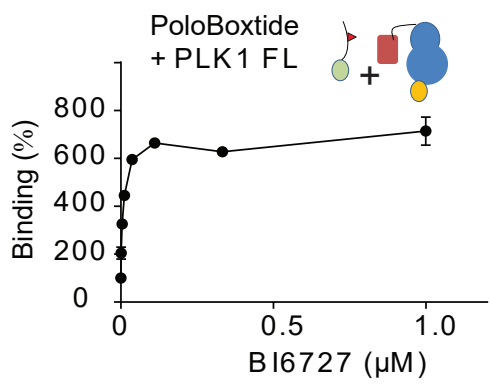

SCR01010<smiles>Cc1ccc(NC(=S)NCCNc2nccc(C(F)(F)F)n2)c(C)c1</smiles>

AW00551

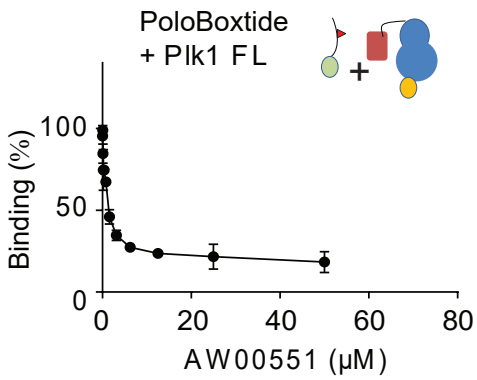

AW00551 
Figure 3

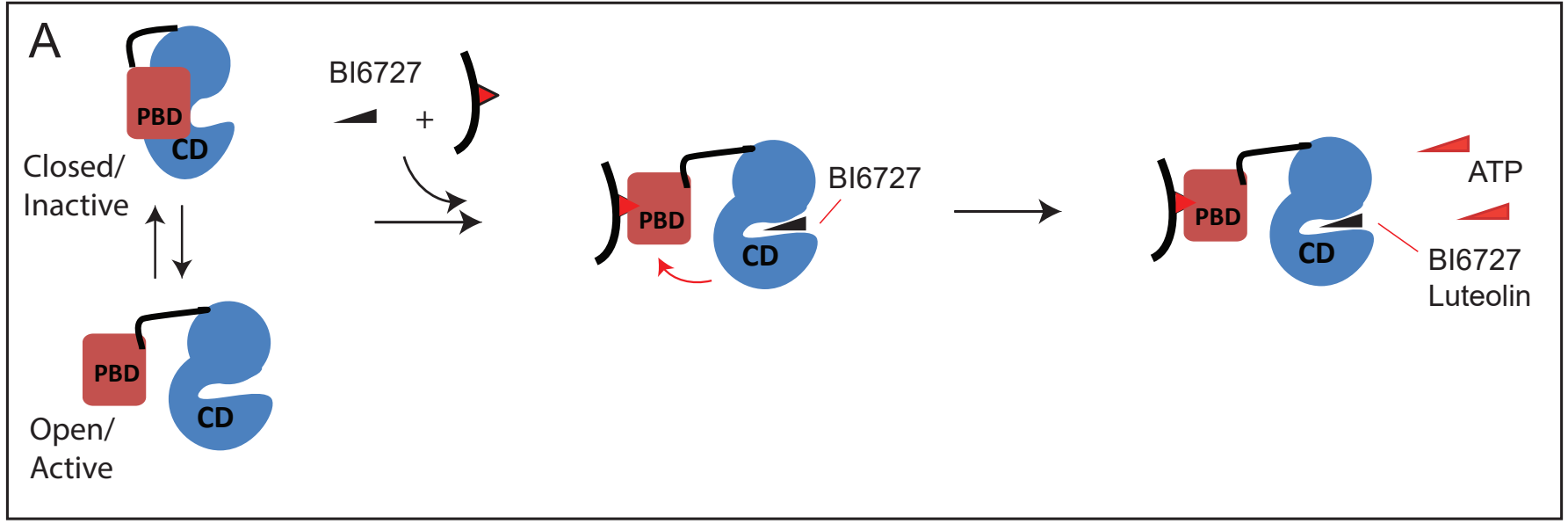

B $\quad$ FM00204
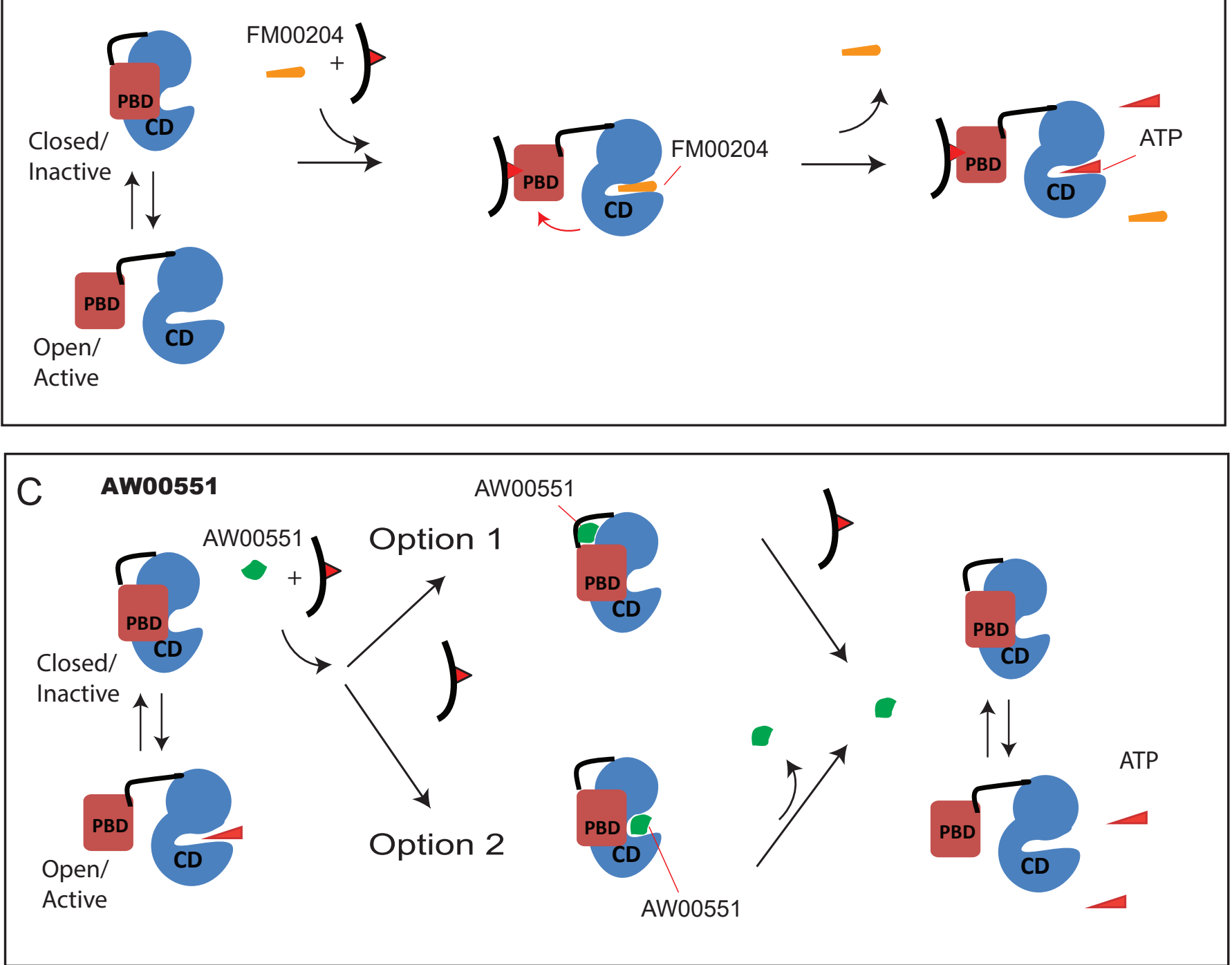
A.
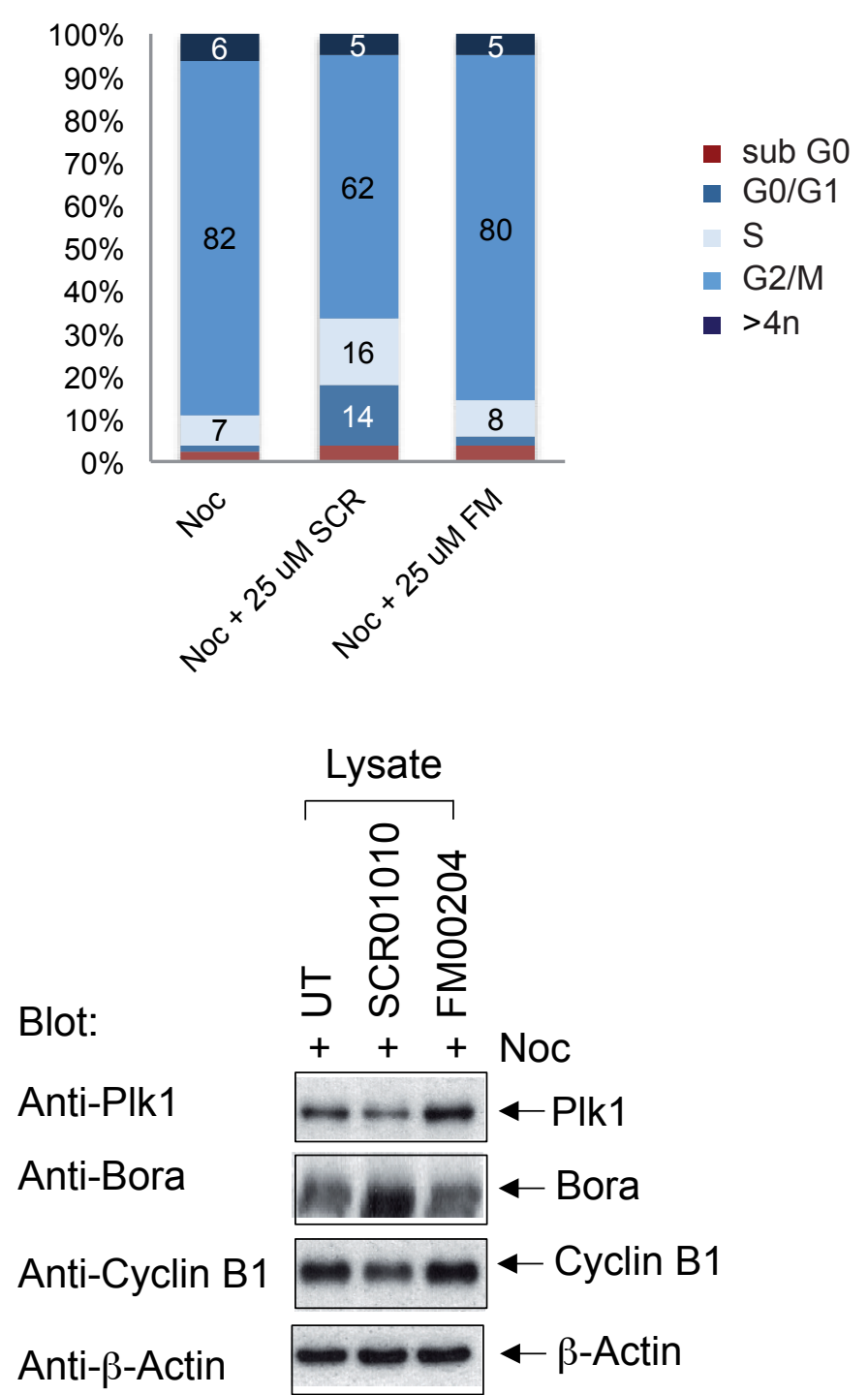

Blot:

Anti-Plk1

Anti-pT210

Anti-Bora
anti-Plk1 IP
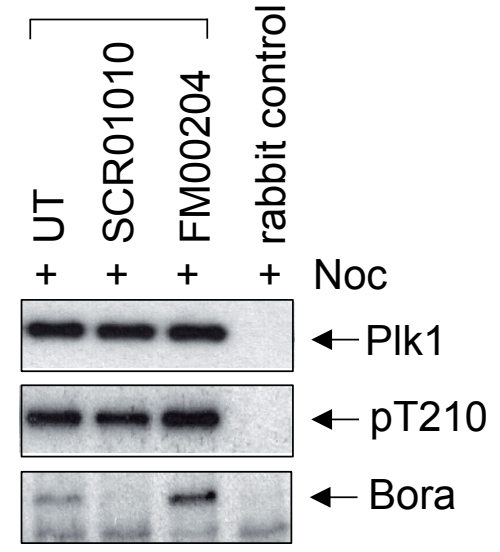

C.

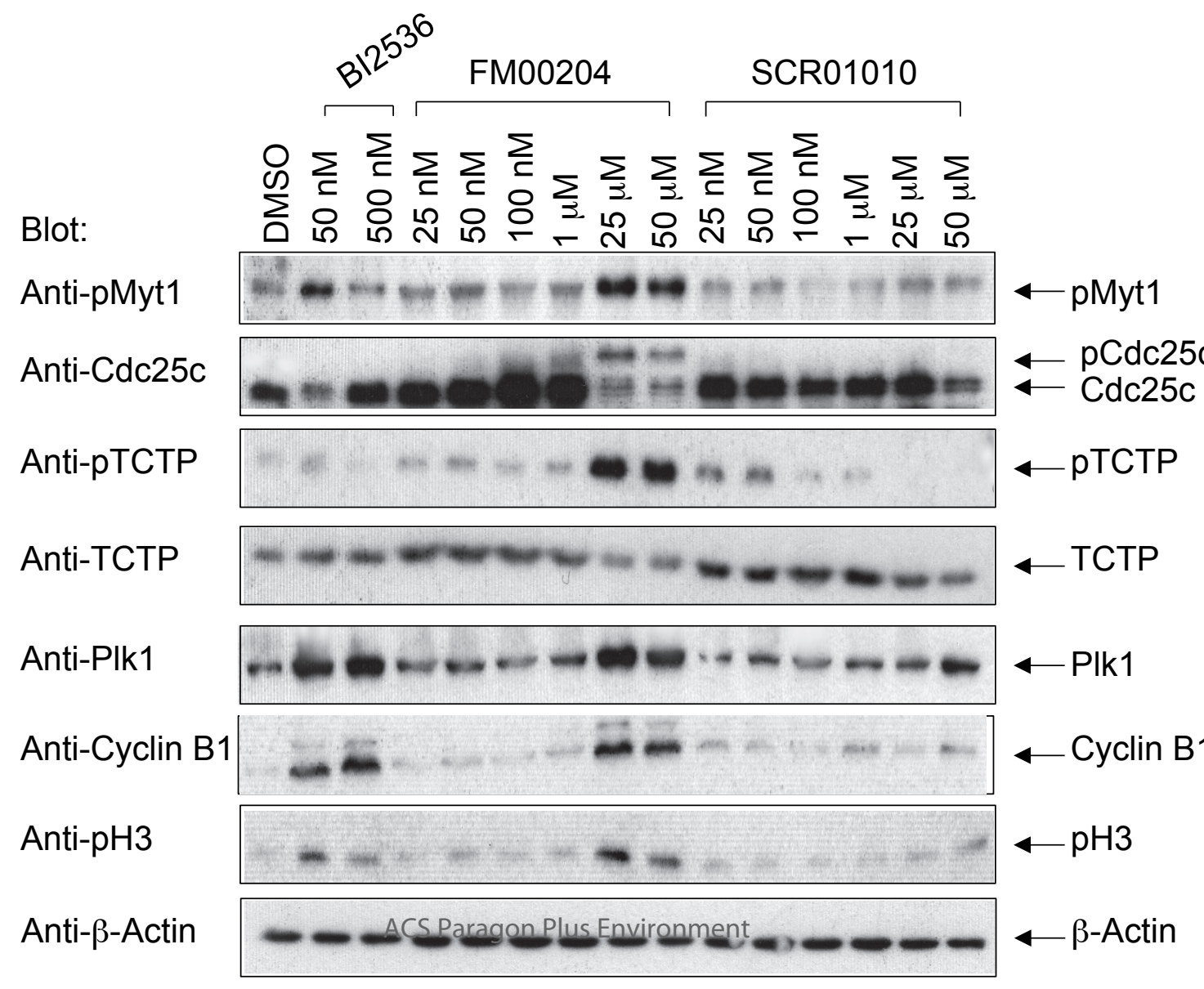




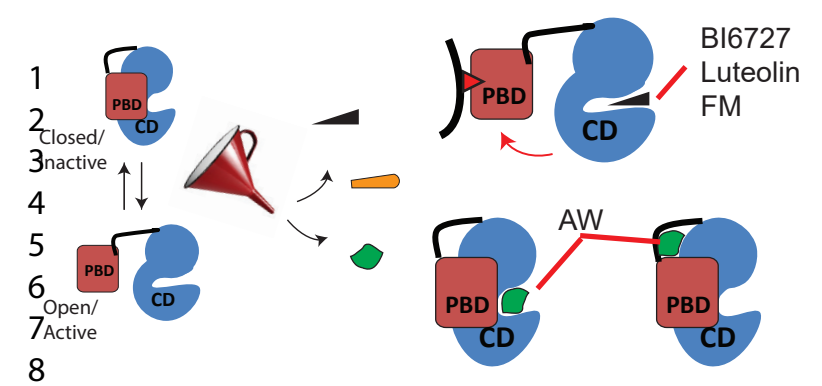

9

10

11

12

13

15

16

17

18

19

20

21

22

23

24

25

26

27

28

29

30

31

32

33

34

35

36

37

38

39

40

41

42

43

44

45

46

47

48

49

50

51

52

53

54

55

56

57

58

59

60 